\title{
On a generalized approach to manufacturing energy efficiency
}

\author{
Apostolos Fysikopoulos • Georgios Pastras • \\ Theocharis Alexopoulos • George Chryssolouris
}

Received: 27 September 2013 / Accepted: 25 March 2014 / Published online: 22 May 2014

(C) Springer-Verlag London 2014

\begin{abstract}
Energy efficiency has become a very significant factor, requiring its inclusion in the manufacturing decision-making attributes. This paper proposes a generalized approach to manufacturing energy efficiency. The basic element of the approach is the division of energy efficiency definition and study into four manufacturing levels, namely process, machine, production line, and factory. Process-level definitions are provided for the majority of manufacturing processes. A machine-level study indicates and solves difficulties, generated by the workpiece geometry, and points out the interaction with the process level through factors, such as the process time. Moreover, machine tool peripherals are responsible for a significant portion of the consumed energy, and classification based on the dependence of their consumption on process variables is required. Studies made on the production line and factory levels show that energy efficiency, at these levels, is heavily dependent on production planning and scheduling and can be improved through the appropriate utilization of machines, with the inclusion of shutdown and eco-modes. Finally, a case study is presented, showing that many of the difficulties towards the optimization of energy efficiency can be dealt with successfully, using the proposed generalized approach.
\end{abstract}

Keywords Energy efficiency · Sustainable manufacturing $\cdot$ Manufacturing processes $\cdot$ Production planning $\cdot$ Scheduling

A. Fysikopoulos · G. Pastras - T. Alexopoulos ·

G. Chryssolouris $(\bowtie)$

Laboratory for Manufacturing Systems and Automation,

Department of Mechanical Engineering and Aeronautics,

University of Patras, 26110 Patra, Greece

e-mail: xrisol@1ms.mech.upatras.gr

\section{Introduction}

Manufacturing is defined as the transformation of materials, energy, and information into goods for the satisfaction of human need [1,2]. Manufacturing processes use one or more physical or chemical mechanisms (Fig. 1) to transform the material's form, shape, or properties [1].

The required energy, as seen in Fig. 1, is considered being a process input. This input is only partially embodied in the final product, in the form of useful work; the rest is transformed into waste heat. Current studies [4] indicate that only a small fraction of the energy input is actually adding value to the product; the majority of the energy is used for creating stable process conditions and peripheral functions.

The manufacturing sector has been historically one of the biggest energy consumers $[5,6]$. Thus, energy efficiency has become a very significant factor, especially in countries that are not energy-independent. Additionally, energy is an increasingly important cost factor due to its rising price. Manufacturing enterprises have to reduce energy consumption for minimizing cost and environmental impact, trying to find new ways to produce "more with less" [2]. Thus, the manufacturing tetrahedron [1] has to be extended as shown in Fig. 2, in order to include energy and eco-efficiency as new attributes for manufacturing decision making. The positioning of energy and eco-efficiency on top of the cost, as well as the red line connecting them in Fig. 2 is not arbitrary. These attributes are the ones that are not connected competitively; on the contrary, to a degree, they have a cooperative relation, as a coincidence of the huge contribution of the energy cost to the total cost.

Although energy efficiency is a relatively new field in manufacturing research, there is already quite an extensive literature, focusing on different levels of manufacturing. Energy efficiency at the process and machine levels for 


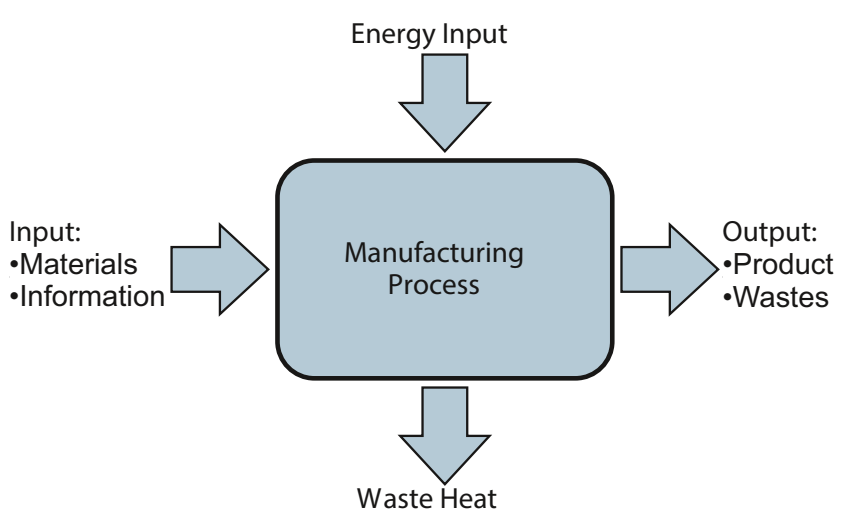

Fig. 1 Process from an energy point of view [3]

conventional processes, such as forming [7-9] or materialremoving processes [9-14], have recently collected some interest. There are fewer studies on nonconventional processes, namely EDM $[15,16]$ or laser machining $[9,17$, 18]. There is also research done on real-time energy consumption measurement $[19,20]$, which is necessary for the implementation of energy-efficient production strategies. Apart from theoretical models of manufacturing processes, there are also some interesting approaches that lead to process energy efficiency optimization through continuous trial and error loops (for which monitoring is a necessary part), such as genetic algorithms [21].

At the machine level, it is also very important that energy consumption is studied regarding the peripheral devices that actually consume a very important part of the energy [4, 22]. The peripheral device consumption can be studied more intuitively with the use of diagrams, introduced by Gutowski et al. in [3] for conventional processes and by Fysikopoulos et al. in [17] for nonconventional processes.

An important issue concerning energy efficiency is that achieving more energy-efficient production may result in worsening of other indicators such as cost or quality.

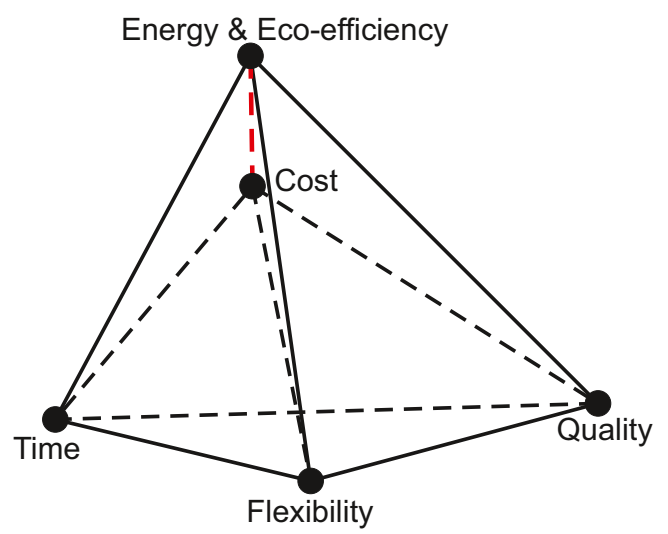

Fig. 2 The manufacturing decision-making attributes with the inclusion of energy and eco-efficiency
However, recent studies propose that energy gains can be achieved without sacrificing a lot at least in terms of quality [14, 23].

It is a fact that a very significant amount of energy is spent on the idle consumption of machines [4]. Thus, energy efficiency at the production line or at factory level can be improved by better production planning and scheduling. An additional advantage of this approach is the fact that there is no interference with indicators such as quality. Several studies propose methods of energy-efficient scheduling that may result in significant energy gains [24, 25]. A necessary element of such an approach is the inclusion of energy efficiency in the parameters predicted by production simulation systems [26, 27]. Other approaches to energy efficiency include life cycle assessment methods [28-30].

In this paper, a generalized approach to manufacturing efficiency is introduced. The main consideration of this approach is the division of energy efficiency analysis into four levels: those of process, machine, production line, and factory.

- Process level: This concerns the energy interactions related to the physical mechanisms of the process itself. For example, in milling, the energy input at the process level is the energy required for the tool to remove the material, while in laser machining, it is the energy of the laser beam.

- Machine level: This includes the process level as well as all the required machine peripherals that ensure proper machining conditions. In some cases, the machine level can be identical with a machine tool; however, special care has to be taken when some peripheral devices are shared among different machines.

- Production line level: This refers to a group of different machines and any other peripherals devices that may be required for the proper function of a production line.

- Factory level: In the same logic as above, this level usually comprises several different production lines that may interact and include peripheral devices, required for the proper function of the entire factory. The latter may have interactions with other factories inside a global production network.

The main issues at each level, as well as the inter-level interactions are analyzed, and a general strategy towards energy efficiency optimization is proposed.

\section{Energy efficiency and manufacturing levels}

Energy efficiency is a metric used for the comparison of different manufacturing configurations, resulting in the selection of the one that produces more with less energy [2]. In order for energy efficiency to be appropriately defined, it 
is of critical importance that the options which are available for comparison are understood.

One first set of options to be compared are the different process variable selections for a given process. The study of these alternatives requires a deep analysis of the process' physics, and it is probably the least studied energy efficiency sector in the existing literature. Subsequently, the different machine tools capable of performing the same operation have to be compared. These machines may use the same or different manufacturing processes. The selection of the most energy efficient machine is correlated not only with the process, but also with the peripheral functions the machine needs to have in order to ensure stable machining conditions.

Moreover, the different production line configurations have to be taken into consideration. Although this problem is conceptually simpler, it is characterized by higher complexity [31,32]. A specific line configuration may be more efficient for a number of reasons, including the ability of better scheduling towards reducing idle times or the inclusion of machines able to switch to eco-mode or shut down when they are not operating. Finally, a last set of options to be compared are the different factory configurations. The possible energy gains from such configurations are of similar nature as for the different production line configurations; however, this is a more complicated problem.

The combination of all information above should allow for the selection of the best possible scenario that optimizes the energy consumption of the entire factory. Energy efficiency targets at optimizing an enormous set of features that can be organized into four groups. This categorization divides the study of energy efficiency into an equal number of different levels (Fig. 3). For each of these levels, the energy efficiency should be defined in an appropriate way, so that the relevant group of objectives described above could be achieved.

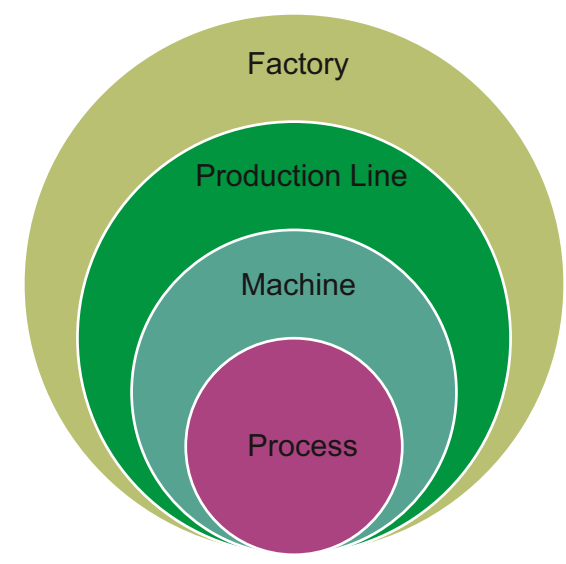

Fig. 3 Energy efficiency analysis division [33]
2.1 Inter-level interactions and energy efficiency optimization strategy

It may seem reasonable that the introduction of these levels can indicate the optimum solution for the entire system in a simple way, the level-by-level optimization of the relevant options via a bottom-up approach (from the process to factory level). It can only be a first, simplistic view of the problem, but it definitely does not provide the optimum solution given that the different levels are subject to nontrivial inter-level interactions. Two simple abstract examples are provided for clarification.

Example 1 (Process-machine inter-level interaction) A given process can be used with two different sets of variables indicated by indexes 1 and 2 . When used with the selection of variables 1 , the required energy for a given task is $E_{P 1}$ and the necessary time $t_{1}$, while when used with the selection of variables 2 , it is $E_{P 2}$ and $t_{2}$, respectively. It is assumed that $E_{P 1}<E_{P 2}$ and $t_{1}>t_{2}$. The selection of the first set of variables is the most efficient at the process level, since $E_{P 1}<E_{P 2}$. However, at the machine level, it is the peripherals' consumption that has to be taken into account. If the consumed power by the peripherals is notated by $P$, then the energy that will be consumed by the machine is equal to $E_{M 1}=E_{P 1}+P t_{1}$ for the first selection of variables and $E_{M 2}=E_{P 2}+P t_{2}$ for the second. Thus, since $t_{1}>t_{2}$, it is not obvious which selection of variables is the most energy-efficient at the machine level. This example indicates an interlevel relation between the process level and the machine level and shows the importance of process time in energy efficiency.

Example 2 (Machine-production line inter-level interaction) Two given machines can be used for the same operation. They are indicated by indexes 1 and 2. For the operation in consideration, it is assumed that the first machine requires energy $E_{M 1}$, while the second one energy $E_{M 2}$, with $E_{M 1}<E_{M 2}$, and the two machines perform the given operation at the same time. At the machine level, the first one is more efficient. However, the two machines are characterized by idle powers $P_{1}$ and $P_{2}$, assumed to obey $P_{1}>P_{2}$. At line level, the energy that will be consumed by each of the two machines is $E_{L 1}=N E_{M 1}+$ $P_{1} t_{\text {idle }}$ and $E_{L 2}=N E_{M 2}+P_{2} t_{\text {idle }}$, respectively, where $N$ is the number of operations performed in the time period studied and $t_{\text {idle }}$ is the idle time. Since $P_{1}>P_{2}$, it is not obvious which of the two machines is the best choice. This example points out an inter-level relation between the machine level and the production line level and shows the importance of idle consumption in energy efficiency. 
This kind of inter-level relations may give the impression that the division of the energy efficiency study into these levels is not very useful. It is true, though, that an attempt for the simultaneous optimization of all the parameters involved, at all levels, presents very high complexity [32, $34,35]$. Even if such an approach is allowed by the current computational systems, the classification of the energy efficiency study into the four aforementioned levels significantly reduces the difficulty of the problem. The inter-level interactions point out that characteristics other than just those of the energy efficiency from each level, namely process time or idle power found in the examples used, should be taken into account when the energy efficiency, at a higher level, is being calculated. Thus, we propose that the energy efficiency optimization strategy should be structured as follows:

1. Study of each process. The outcome should be a model relating the process variables to the energy efficiency, at the process level, besides relating the process variables to other quantities, such as process time, that may alter the energy efficiency at the machine level.

2. Comparison of the different machines available for each necessary operation. Each machine should be compared when using a variety of process variables. The output should be as follows:

(a) The appropriate process variables for each machine that maximizes the energy efficiency at the machine level.

(b) A table relating the machine selection to the energy efficiency, at the machine level, but also relating the machine selection to other quantities that may alter the energy efficiency, at the production line level, such as idle power.

3. Comparison of the different production line configurations. The output should be as follows:

(a) The appropriate machine for each operation that maximizes the energy efficiency at the production line level.

(b) A table relating the production line configuration selection to the energy efficiency at the production line level, but also relating the production line configuration selection with other quantities that may alter the energy efficiency at the factory level.

4. Comparison of the different factory configurations. The output should be as follows:

(a) Selection of the best alternative for each production line.

(b) Selection of the best alternative for the factory.

\subsection{Energy efficiency definition}

Useful energy outputs may be measured by a variety of thermodynamic or physical indicators, the appropriate choice of which will depend upon the system under consideration, the purpose of the analysis, and the availability of the relevant data $[36,37]$.

For each of the levels described above, the energy efficiency metric has to be defined. A simple selection is the energy consumption itself. However, the energy consumption should be compared with the output generated by the use of this energy. If simply the energy is used as a metric, then the obvious optimum solution is no production, no energy consumption.

Energy is not an appropriate quantity to be used, since it is an extensive quantity, a quantity proportional to the system's size [38]. For the same reason that energy is not appropriate for the comparison of two thermodynamic systems and the decision as to which one is hotter, since the energy is proportional to the size of the system, then the energy cannot be considered appropriate for the comparison of the energy efficiency of two factory configurations. For this reason, in thermodynamics, temperature is defined as an intensive quantity that does not depend on the size of the system being considered. Similarly, energy efficiency has to be defined as an intensive quantity.

We propose that energy efficiency is defined as the quotient of the process's result measure over the energy required for the acquisition of this result

$$
\text { Eef } \equiv \frac{\text { result }}{\text { energy required for the result }} .
$$

Since energy is an extensive quantity, the "result" has also to be an extensive quantity, so that the demand that energy efficiency is defined as an intensive quantity is satisfied.

Definition (2.1) may appear static due to the absence of the process time. However, it has to be noted that the "energy required for the result" is a function of the process variables and, thus, a function of the process time. Since most of the processing devices have some minimum idle consumption, it is true that the consumed energy and consequently, the energy efficiency, present a strong dependence on the process time $[9,33]$.

If the "result" in Eq. 2.1 is selected as a quantity with energy units, the energy efficiency is defined to be dimensionless. For this approach, a suitable candidate for the numerator of Eq. 2.1 may be the theoretical minimum energy required for the same "result" [3, 39-41]. Then, energy efficiency has the natural interpretation of the percentage of the total energy provided to the system that gets transformed into useful work. This kind of definition, although it can be implemented for a specific single process, 
is difficult to be generalized for the entire class of processes, given that the theoretical minimum energy for the required "result" may be different for each kind of process. Thus, such definition may not be appropriate for the energy efficiency that has to be used as a comparative measure between different processes. Additionally, the production line and factory levels "results" could not be defined on this basis since many different processes are involved. However, it can be an interesting and useful approach only at the process level. Due to the inter-level interactions, described in Section 2.1, such an approach is not followed in this study.

Therefore, a careful definition of the "result" and the "energy required for the result" is necessary in order for the defined energy efficiency to serve the purposes described above and allow the strategy of Section 2.1 to be followed. The denominator of the energy efficiency definition (2.1) is easier to be defined than the numerator. At each level, an amount of energy is entered as an input. A part of this is wasted, and the rest is transferred to the consequent lower level or is transformed into useful work, if the process level is considered.

\section{Example 3 (Energy transfers between manufacturing} levels) A production line is assumed to be composed of a milling machine and a laser cutting machine. Some amount of energy $E_{L}$ is consumed by the entire line. In this example, the milling machine is performing its task faster than the laser cutting machine does. Thus, the milling machine remains idle for some amount of time. A percentage of the energy consumed by the production line is wasted on the idle consumption of the milling machine. The rest is used by the machines to produce useful work.

The laser cutting machine is consuming some amount of energy $E_{M}$. A percentage of this energy is consumed in peripheral functions of the machine, dedicated to stabilizing machining conditions, such as the laser machine cooling unit [17]. The remaining energy becomes the energy of the laser beam that is the input energy of the process $E_{P}$. At the process level, more losses will accrue, and in the end, only a percentage of $E_{P}$ is going to be transformed into useful work.

The example suggests that the denominator in energy efficiency definition (2.1) should be different at each level and specifically as follows:

1. At the factory level, the denominator should be $E_{F}$, the energy consumed by the factory.

2. At the production line level, the denominator should be $E_{L}$, the energy consumed by the production line.

3. At the machine level, the denominator should be $E_{M}$, the energy consumed by the machine.
4. At the process level, the denominator should be $E_{P}$, the energy input of the process.

In an obvious way, it is always true that

$E_{P}<E_{M}<E_{L}<E_{F}$.

Figure 4 explains the above inequality in a graphical way.

According to what has already been stated, the "result" has to be a quantity proportional to the size of the production that enables the comparisons described in this section.

The process-level energy efficiency should serve as a comparative metric between the selections of different process variables. A study of the process efficiency should be generic and useful for all possible applications of the specific process. Thus, the "result" of the process has to be strongly connected to the physical transformation that it exerts on the workpiece. There is a need for the manufacturing processes to be classified in groups, where the "result" is similar. Then, the "result" should be defined for each of the classes. This is the main purpose of Section 3.

An appropriate selection for the machine-level "result" could be the same as that of the process level, but this is not always true. As it will be further analyzed in Section 4, in material removing operations, the appropriate machinelevel "result" has to depend on the geometry of the workpiece, in order to ensure that machine-level energy efficiency serve as a comparative measure between machines able to perform the same operation.

At the production line and factory levels, things are more complicated. A production line may perform a series of different operations that are incomparable and exert a series of different characteristics (machine-level results) that cannot be added in any sense. At the production line level, the unique measure that can be used for the quantification of

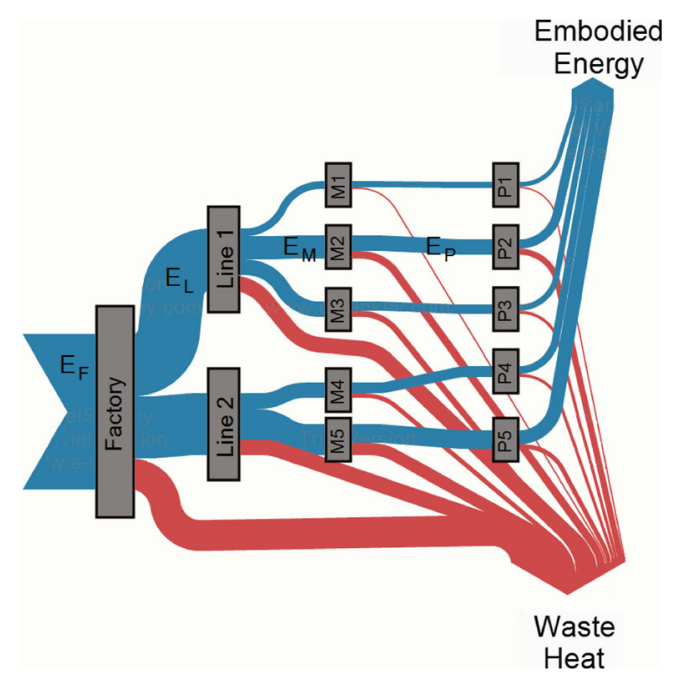

Fig. 4 Energy interactions between the different levels 
the output that preserves the wanted properties is the number of products produced. A reasonable question is how this should be modified in case that a production line or a factory produces more than one kind of different products. In this case, the energy efficiency has to be defined for each kind of products.

\section{Energy efficiency at the process level}

Based on Eq. 2.1, the energy efficiency at the process level is defined as

Eef $f_{P}=\frac{\text { result }_{P}}{E_{P}}$,

where $E_{\mathrm{P}}$ is the energy provided to the process. The result $P$ has to be further considered, since manufacturing processes are broadly classified into six categories: primary forming, deforming, removing, joining, coating, and modifying material properties processes [1]. The last class of processes will not be studied here because of its high complexity. As energy efficiency should serve as a comparative measure, at least for different processes within the same class, the "result" has to be defined in a universal way for each class of processes.

At primary-forming processes, the work necessary for the process is approximately proportional to the volume of the body formed. For example, in casting, the major portion of the necessary energy is the energy required to melt the material to be cast, which is proportional to the volume of the formed body. Thus, an appropriate definition for the "result" in primary forming processes is

result $_{P F P} \equiv V_{\text {formed body }}$.

In deforming processes, the "result" has to measure the degree of deformation imposed on the workpiece by the process. The deformation is quantified by the strain tensor defined as

$\mathbf{E}=\frac{1}{2}\left[\left(\nabla_{\mathbf{x}} \mathbf{u}\right)+\left(\nabla_{\mathbf{x}} \mathbf{u}\right)^{T}+\left(\nabla_{\mathbf{x}} \mathbf{u}\right)\left(\nabla_{\mathbf{x}} \mathbf{u}\right)^{T}\right]$,

where $\mathbf{u}$ is the displacement field and $\mathbf{x}$ the position vector. Most certainly, the strain tensor itself is not an appropriate quantity to measure the process "result" as it is not a scalar quantity. There are several scalar quantities that can be produced by the strain tensor in such a way that are coordinate transformation invariants. Typically, the invariant

$I=\frac{1}{2}\left[\operatorname{tr}\left(\mathbf{E}^{2}\right)-(\operatorname{tr} \mathbf{E})^{2}\right]$

is used as a measure of the magnitude of deformation. Then the appropriate "result" in deforming processes is

result $_{D P} \equiv \int I d V$
This "result" is proportional to the deformed volume, because of the volume integral, but also is proportional to the magnitude of the deformation imposed at this volume.

Example 4 (Process result in deforming processes) A clarifying example can be provided by considering the bending of a rod of a given length. Figure 5 shows the initial state of the rod and two possible outcomes of the process.

For each outcome, the invariant $I$ is plotted, and the total "result" is compared. This example also shows that the use of the deformed volume as the "result" is not appropriate, since in both cases, the "result" would be the same, namely the entire length of the rod.

For most removing processes, a good measure for the "result" is the volume of the material removed.

result $_{R P} \equiv V_{\text {removed }}$

However, in dividing processes, (e.g., shearing), there is no removed volume. Since in these cases, only the bonds on the separated surface are altered during the process, an appropriate quantity for the measuring of the magnitude of the process "result" is the area of the surface created by the process

result $_{R D P} \equiv A_{\text {created }}$

In most joining processes, a good measure of the "result" is the volume of the joint created. Thus,

result $_{J P} \equiv V_{\text {joint }}$

This definition may present inflexibility when external materials, such as glues, are used in order for the joint to be created. However, these cases present little interest in terms of energy consumption. For all energetically interesting cases, such as resistance spot welding or laser welding [42], this definition is a good comparative measure. It has to be

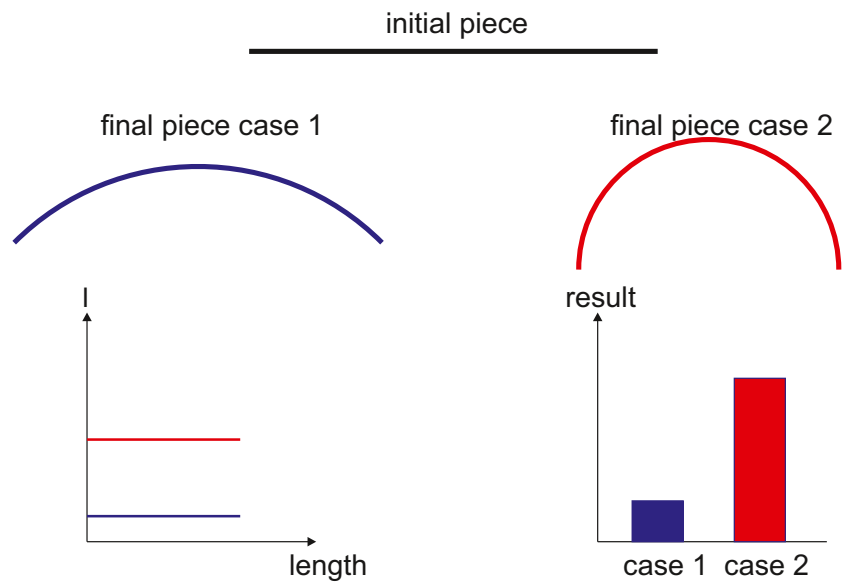

Fig. 5 An indicative example regarding the "result" in the case of bending 
noted that a good weld is not necessarily a large weld; there is always an optimum size, yet the incorporation of other criteria, such as quality, can resolve this issue.

In coating processes, the process outcome can be considered being proportional to the coated surface and the depth of the coating. In other words, the "result" can be defined to be the coating volume

result $_{C P} \equiv V_{\text {coating }}$

To sum up, the volume that is affected by the process, in most cases, is the appropriate selection for the process "result." In deforming processes, the "result" is not defined as the deformed volume; however, it is proportional to it.

Following this approach, an energy efficiency library for each process can be constructed, since the process-level energy efficiency has been defined in a way that depends neither on geometrical or other details regarding the workpiece nor on the details of the machine performing the operation. This library will connect the process variables with the energy efficiency at the process level, based either on modeling or on experiments and can be the basis for the study of energy efficiency at the higher levels, aiding the manufacturing community towards achieving higher energy efficiency.

\section{Energy efficiency at the machine level}

Many studies have shown that the actual consumed energy, required for machining processes is much exceeded by the energy demand of the related peripheral equipment. These comprise coolant pumps, lubrication supply and compressed air supply that perform the auxiliary processes [33, $42,43]$. Studies show that less than $13 \%$ of energy expenditures have been utilized for productive operations [44].

Based on Eq. 2.1, the energy efficiency at the machine level is defined as

$$
E e f_{M}=\frac{\text { result }_{M}}{E_{M}}
$$

where $E_{M}$ is the energy provided to the machine. The result $_{M}$ is defined and further discussed in Section 4.2.

\subsection{Machine peripherals' consumption}

Since the peripherals are responsible for a significant part of a machine's consumption [4, 17], they should be studied in detail. An important characteristic of each of the peripherals' consumption is its dependence on the process variables. Several peripherals, such as CNC controllers, consume a specific power that does not depend on the working mode of the machine. On the other hand, the consumption of other peripherals, such as cooling units, depends on the selection of process variables in an obvious way. Thus, a first step towards achieving the goals of the study of peripherals is their classification into the two aforementioned classes. Then, the next step is the specification of the dependence of the consumption of the second class on the process variables.

The above classification and the consumption dependence on the process variables can be summarized into the form of diagrams, which were introduced in [3] and were extended and applied to nonconventional processes in [17]. It is advantageous to plot the consumption of the peripheral devices versus the power used at the process, instead of the process variables themselves, in order for the better visualization of the various consumptions as a percentage of the power input to the machine. Figure 6 shows an example of such a diagram.

\subsection{Definition of machine-level result}

For removing processes, the process-level "result" may not be appropriate for the study of energy efficiency at the machine level. This phenomenon is only applicable when an operation removes a segment of the workpiece, since it is not necessary that the entire volume be physically modified to be removed, but only a part of it containing the entire boundary of the volume to be removed.

Example 5 (The need for the introduction of a different definition of the machine-level "result" in removing processes) A machine has to remove a disk from a metal sheet. A milling machine, a laser cutting machine, or a punching machine can be used. The first two machines perform material-removing processes; thus, it looks like the "result" has to be defined as the same quantity. However, as shown in Fig. 7 , the volume of the material that is physically altered

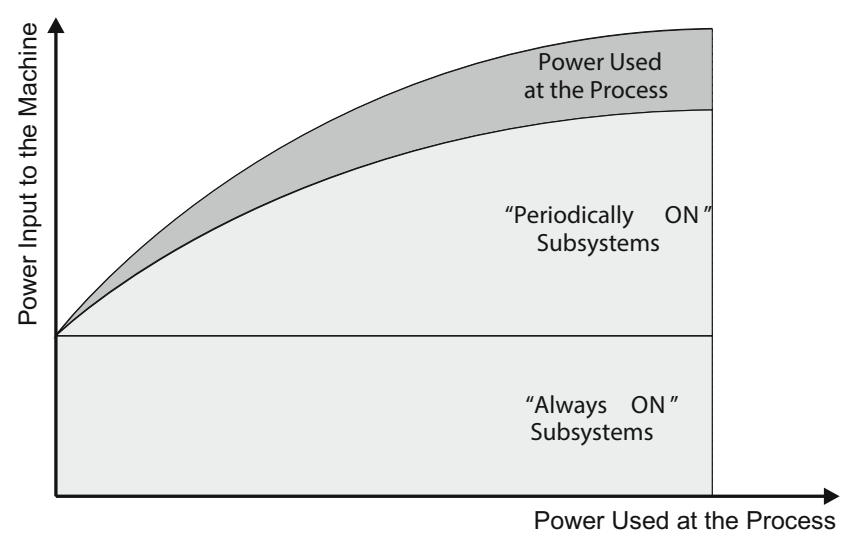

Fig. 6 An indicative diagram describing the classification of the peripheral devices and the dependence of their consumption on the power used at the process 


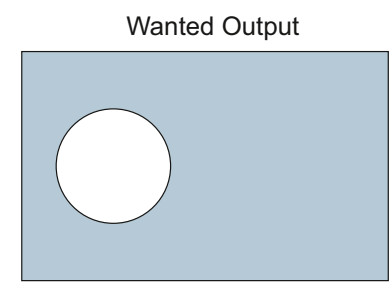

Milling Machine

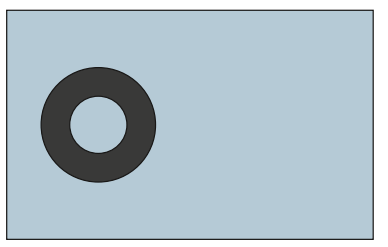

Fig. 7 A removal of a disk from a metal sheet with milling or laser cutting. The dark ring is the portion of the sheet that is physically altered by the process. The problem of definition of the appropriate "result" is apparent

by the specific processes and consequently the process-level "result" is different, since the milling machine diameter and the laser beam diameter are of different sizes.

Although the amount of material physically altered by the two processes is different, the final output, a metal sheet with a circular hole, is exactly the same. The machinelevel "result" has to be considered the same for both cases, so that the two machines can be compared. Furthermore, if the use of the punching machine is considered, since it belongs to the class of separating processes, the processlevel "result" does not even have the same dimensions as the process-level "results" of the other two machines. Indeed, in the punching process, the most natural selection of the process-level "result" is the generated area by the process, as described in Section 3, namely the circumference of the circle multiplied with the sheet thickness. So, the process-level "result," as in the case of the example, should be translated into the machine-level "result," which may depend on the geometrical features of the process, like the tool diameter.

Thus, the machine-level "result" is defined as the process-level "result"

result $_{M} \equiv$ result $_{P}$

for all processes, except for the removing processes, where the machine-level "result" is defined as

$\operatorname{result}_{M} \equiv V_{\text {removed }}$,

where it has to be noted that $V_{\text {removed }}$ includes all the removed volume, either physically altered by the process or not.

It is not possible for the machine-level "result" to be adopted as the appropriate definition for the process level, too. When studies on a specific process are performed, it is highly constraining to define the "result" as something that depends on the geometrical features of a specific product. Energy efficiency, at the process level, should be globally defined and be available in the literature in a way that can be easily translatable into machine-level efficiency. Indeed the translation between the two is not difficult. The same example as before can be clarifying.

Example 6 (Clarification of the relation between the process-level and machine-level "results" in removing processes) In the following, the radius of the disk to be removed is considered being equal to $R$, the laser beam diameter is equal to $d_{\mathrm{l}}$, the milling tool diameter is $d_{\mathrm{m}}$, and the thickness of the metal sheet is equal to $w$. In the case of the laser cutting machine, the "result" at the process level is the volume altered by the laser; thus, result $P$ laser $=$ $\pi\left(R^{2}-\left(R-d_{l}\right)^{2}\right) w$, in the case of the milling machine, it is result $P_{\text {mill }}=\pi\left(R^{2}-\left(R-d_{m}\right)^{2}\right) w$, and in the case of the punching machine, it is result $P$ punch $=2 \pi R w$. In all cases, the machine-level "result" is considered being the removed volume of the entire disk, thus result ${ }_{M}=\pi R^{2} w$. The energy efficiency at the process and machine levels are connected as follows:

$$
\begin{aligned}
& E e f_{M \text { laser }}=\frac{R^{2}}{R^{2}-\left(R-d_{l}\right)^{2}} \frac{E_{P \text { laser }}}{E_{M \text { laser }}} E e f_{P \text { laser }}, \\
& E e f_{M \text { mill }}=\frac{R^{2}}{R^{2}-\left(R-d_{m}\right)^{2}} \frac{E_{P \text { mill }}}{E_{M \text { mill }}} E e f_{P \text { mill }}, \\
& E e f_{M \text { punch }}=\frac{R}{2} \frac{E_{P \text { punch }}}{E_{M \text { punch }}} E e f_{\text {Ppunch }},
\end{aligned}
$$

where $E_{P}$ and $E_{M}$ are the energies consumed by the process and the machine, respectively. The advantage of this approach is the fact that $E e f_{P \text { laser }}, E e f_{P \text { mill }}$, and $E e f_{P \text { punch }}$ can be provided in the literature. The only complexity introduced is the inclusion of the simple geometrical factors in formulas (4.4), (4.5), and (4.6).

\section{Energy efficiency at the production line level}

Energy management defines the sum of all processes and measures in order for minimal energy consumption to be ensured by a given production demand, including the implementation of organization, information structure, and tools [45]. Considering that both operations and possible improvement measures are usually implemented at the single process level, the calculation of energy efficiency should be integrated into the bottom layer and then be transferred to the upper levels through information technology (IT) systems [46].

All the above clearly indicate the need for the assessment of energy demand of production systems, considering 
the dynamic behavior of machines. The dynamic load profile of a manufacturing process can be divided over time by energy analysis. Such analysis clearly suggests that all defined operating states of the given process are varying in their mean power demand, timing, and therefore also in their energy amounts. The application of electricity control policies and tariffs has further complicated the system's optimization scheduling problem [31], since an optimized plan that leads to reduction in electricity consumption does not necessarily lead to reduction in the electricity cost. However, very little research [27, 31, 42] currently focuses on this problem, though it is important that the trade-off between electricity consumption reduction and cost saving be delivered.

In the decision-making hierarchy of the production planning and control function of manufacturing systems, detailed scheduling constitutes the final step before the actual output occurs. Scheduling encompasses allocating workloads to specific work centers and determines the sequence in which operations are to be performed. Generally, the objectives of scheduling are that trade-offs be achieved among conflicting goals, comprising efficient utilization of staff, equipment, facilities, and minimization of customer waiting time, inventories, and cycle times. Nearly every manufacturing organization has to deal with scheduling tasks on a daily basis. Nevertheless, although the academic research has generated an abundance of theoretical work, on a number of classical scheduling problems, the use of academic results in industry has been rather minimal [1]. A particular reason for this fact is that the actual manufacturing systems are extremely variable, and many academic approaches are far from practical when used in an industrial environment. Overall, the complexity of the scheduling problem [31] and the increasingly important need for the optimization of a manufacturing system's performance in today's competitive world dictate the formulation of new, practical, ready-to-use methods in order for the production scheduling challenges to be addressed.

An apparent problem in the production line level is the determination of the appropriate "result" to be used in the energy efficiency definition. This issue is the outcome of the fact that a production line performs several different operations that add value to the final product in very different and incomparable ways. Each machine of a production line contributes a machine-level "result," but it is not possible for these "results" to be added for the acquisition of a production-level "result." At the production line level, the measure that quantifies the output is the number of products manufactured:

$$
E e f_{L} \equiv \frac{N_{L}}{E_{L}}
$$

where $N_{L}$ is the number of products manufactured, and $E_{\mathrm{L}}$ is the amount of energy consumed by the production line for the manufacturing of these products.

Operational methods including genetic algorithms, dispatching rules, and adaptive search procedure to minimize the electricity consumption and classical scheduling objectives on a single machine and on parallel machines have been investigated in several works [42, 47, 48]. These investigations should be based upon the observation that in manufacturing environments, large quantities of energy are consumed by nonbottleneck machines as they lay idle. A simple thought could be that such machines should be turned off when idle. However, typically, there is some startup energy consumption that makes the option of turning off the machine profitable only when it is applicable for time intervals larger than a critical point as shown in Fig. 8. For smaller time intervals, an intermediate eco-mode may be preferable. In such a mode, all noncritical peripherals should be turned off. Machine tool producers can help by developing machines with such features.

\section{Energy efficiency at the factory level}

Studies [3, 42, 49] for the use of energy in production show that different production rates generate different levels of energy consumption. The energy efficiency of the entire factory system is not derived directly from the sum of individual parts or actions. Only through a holistic view of the complex coherences and interactions of individual resources, processes, and structures of a factory can energy optimization potentials of the overall process or the total system be exploited [42, 50].

Production planning plays a significant role on the energy efficiency of a factory. The load management of the line can offer great savings from an energy point of view. Moving

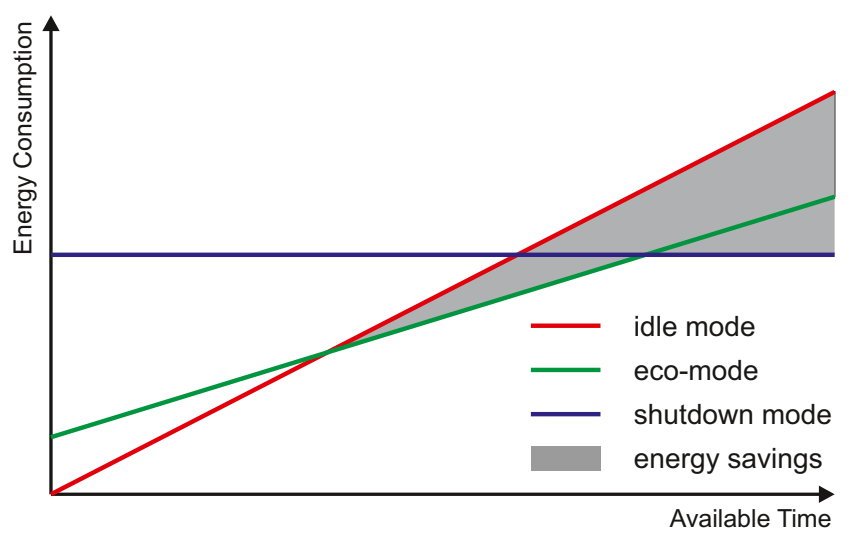

Fig. 8 An indicative diagram for the energy consumption of a machine during nonworking time periods under different available modes 
towards an energy-efficient production planning requires the inclusion of energy and eco-efficiency within the goals of production design and control at all levels, together with time cost, quality, and flexibility (Fig. 2). The reduction of the idle time through energy-efficient process planning with the combination of better batch and order organization will lead to a better prediction of the workflow. The forecasting of the requested orders is released, and lot sizes can provide the production manager with adequate information for the even time distribution of the load in order to prevent stalling and leaving the resources idle.

In a simplistic case of a factory constituted by independent production lines, the factory energy efficiency optimization is identical with the optimization of the energy efficiency of each of the lines. However, in most cases, production lines interact with each other. The most energyefficient selection will emerge through the appropriate production planning and scheduling. Several tools have already been developed for this purpose [51, 52]; nevertheless, they need to be upgraded with the inclusion of energy efficiency in their decision-making arguments [53].

The definition of energy efficiency at the factory level needs to consider that several different kinds of products that may be produced. In this case, questions may arise when a change in the factory configuration leads to the increase of the energy efficiency for one kind of product and to the reduction for another. In order for such questions to be answered, the overall energy efficiency at the factory level has to be defined. First, energy efficiency has to be defined for every kind of products. At this definition, the "result" is again considered as the number of manufactured products:

Eef $_{F, i} \equiv \frac{N_{F, i}}{E_{F, i}}$,

where $N_{F, i}$ is the number of the products identified with index $i$ manufactured, and $E_{F, i}$ is the amount of energy consumed by the factory for the manufacturing of these products. The overall factory-level energy efficiency has to be a weighted average of the energy efficiencies for each product, since it is the products requiring more energy to be manufactured that have a greater influence on the overall energy efficiency. From the above statement, a reasonable selection for the weight is the energy required to manufacture the relevant product at the factory level $E_{F, i}$. In this case, if $N_{i}$ products of the $i$ kind are to be manufactured, and the overall energy efficiency equals

Eef $_{F}=\frac{\sum_{i} N_{i} E_{F, i} E_{e f}, i}{\sum_{i} N_{i} E_{F, i}}=\frac{\sum_{i} N_{i} \text { parts }_{i}}{E_{F}}$.

This definition is again of the general form (2.1), where the "result" can be considered the collection of $N_{i}$ products of each kind $i$.
Similar shutdown techniques can be applied to the production lines that constitute the factory, as applied to the machines in a production line, in order for the idle consumption to be reduced and the factory energy efficiency to be increased.

\section{Case study}

\subsection{Case study description}

A case study has been implemented to show the application of the energy efficiency optimization strategy presented in Section 2.1 and the definitions for energy efficiency presented in Sections 3, 4, 5, and 6. The case study was about the making of two through holes with circular cross section with diameters 4.9 and $22 \mathrm{~mm}$ in a mild steel sheet of thickness $w=2 \mathrm{~mm}$ (Fig. 9).

Two processes, drilling and abrasive water-jet machining (AWJM), are compared. The drill cannot open a wide hole in one operation, but it has to widen the holes in steps of step $_{\text {drll }}=5 \mathrm{~mm}$ at most. It has to be noted that, unlike the drilling process, the AWJM is a trepanning process. A setup time for the proper mounting is required before each operation. For the drilling process, the user can select the feed rate, and for the AWJM process, the pump pressure.

\subsection{Process-level study}

The cutting force $\left(F_{c z}\right)$ per tool edge [54] equals

$F_{c z}=\frac{d_{1}-d_{2}}{2} s_{z} k_{\varphi} k_{s t 1}\left(s_{z} \sin \frac{\omega}{2}\right)^{-z}$,

where

$d_{2} \quad$ is the predrilling diameter,

$d_{1} \quad$ is the finish and the tool's diameter,

$s_{z} \quad$ is the feed per revolution and tool edge (ranging between 0 and $0.1 \mathrm{~mm} / \mathrm{rev}$ ),

$\omega \quad$ is the point angle of the twist drills $\left(160^{\circ}\right)$,

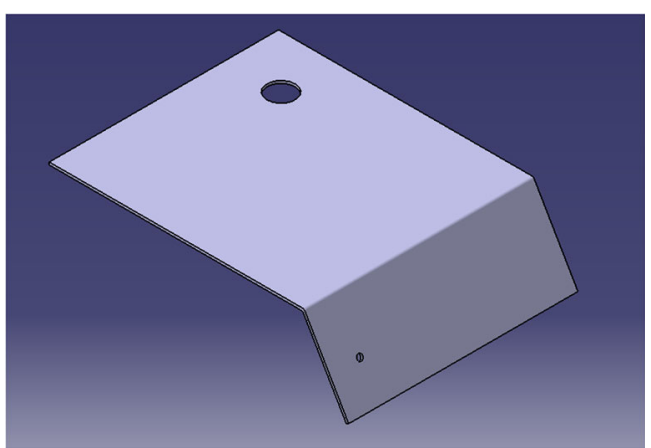

Fig. 9 The workpiece 
$k_{\phi} \quad$ is a correction factor taking into account tool wear (1.4),

$k_{s t 1} \quad$ (specific cutting force) is material-dependent,

$z \quad$ (exponent) is material-dependent.

For mild steel, $z=0.17$ and $k_{s t}=178 \mathrm{daN} / \mathrm{mm}^{2}$, if $s_{z}$ is measured in millimetres per revolution and per tool edge.

The corresponding torque $\left(M_{c}\right)$ is equal to

$M_{c}=z_{E} F_{c z} \frac{d_{1}+d_{2}}{4}$,

where $z_{E}$ is the number of tool edges.

The required power $\left(P_{c}\right)$ equals

$P_{c}=2 \pi n M_{c}$,

where $n$ are the drill tool's revolutions per unit time (1,000 rev/min).

The material removing rate $\left(M R R_{\text {drill }}\right)$ can be estimated as

$M R R_{\text {drill }}=\pi \frac{d_{1}^{2}-d_{2}^{2}}{4} z_{E} s_{z} n$

Thus, the process-level energy efficiency $\left(E e f_{P, \text { drill }}\right)$, according to the definition given in Section 3 is equal to

$\operatorname{Eef}_{P, \text { drill }}=\frac{\left(s_{z} \sin \frac{\omega}{2}\right)^{z}}{k_{\varphi} k_{s t 1}}$

The energy efficiency, as a function of the selectable process variable, is shown in Fig. 10.

The time required for the process $t_{\text {drill }}$ ) equals

$t_{\text {drill }}=\frac{w}{s_{z} z n} \operatorname{int}\left(\frac{D}{\text { step }_{\text {drill }}}+1\right)+t_{\text {change }} \operatorname{int}\left(\frac{D}{\text { step }_{\text {drill }}}\right)$,

where

$D \quad$ is the final hole diameter,

$w \quad$ is the workpiece thickness measured in millimetres $(2 \mathrm{~mm})$,

step $_{\text {drill }}$ is the maximum widening of the hole diameter that can be performed with one tool,

$t_{\text {change }} \quad$ is the required setup time of the driller tool, and

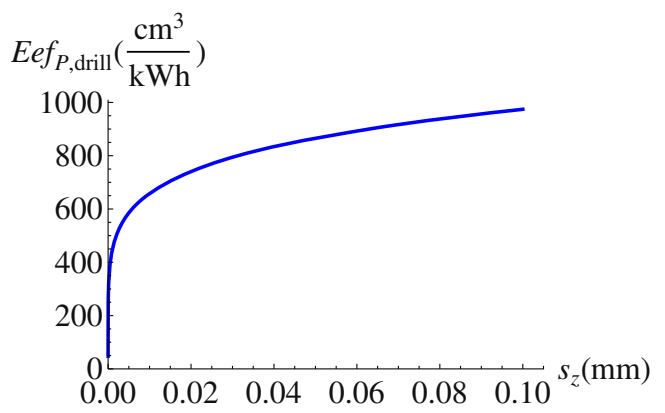

Fig. 10 The process-level energy efficiency for the drilling process as function of the feed rate per revolution per tool tooth int returns the integer part of its argument.

For the abrasive water-jet process, the model used is described in [55]. In this model, the cutting speed is given by

$v_{c}=\left(\frac{f_{a} N_{m} p^{1.594} d_{\mathrm{o}}^{1.374} M_{a}^{0.393}}{c q w d_{m}}\right)^{1.15}$.

$f_{a}$ is dimensionless and describes the abrasive factor, which is used to account for the differences in cutting speeds, due to the use of different abrasive materials. In this study, it is considered equal to 1 (Garnet abrasive),

$N_{m}$ is the material machinability that is dimensionless. For the mild steel, it equals 87.6,

$p \quad$ is the water pressure measured in megapascals (170$320 \mathrm{MPa})$,

$d_{o}$ is the orifice diameter measured in millimetres $(0.4 \mathrm{~mm})$,

$d_{m} \quad$ is the diameter of the mixing tube measured in millimetres $(0.9 \mathrm{~mm})$,

$M_{a}$ is the abrasive mass flow rate measured in grams per minute (575 gr/min),

c is a constant determined by the unit system used, equal to 788 for the units used,

$q$ is the quality level index taking values from 1 to 6 , being equal to 3 in the studied case, and

$w$ is the workpiece thickness measured in millimetres (2 mm).

The material removing rate $\left(M R R_{\mathrm{AWJM}}\right)$ equals

$M R R_{\mathrm{AWJM}}=v_{c} k w$,

where $k$ is the kerf $(1.0 \mathrm{~mm})$.

The water flow rate $\left(f_{w}\right)$ is given by

$f_{w}=\frac{\pi d_{o}^{2}}{4} \mu \sqrt{\frac{2 p}{\rho}}$

where

$\rho$ is the water density $\left(1,000 \mathrm{~kg} / \mathrm{m}^{3}\right)$ and

$\mu$ is the momentum transfer factor (typically 0.75 ).

The power carried by the water flow $(P)$ equals

$P=p f_{w}$

Thus, for the AWJM process, the energy efficiency at the process level ( $E e f_{P, \text { AWJM }}$ ) equals

Eef $_{P, \mathrm{AWJM}}=\frac{2 k w}{\pi d_{\mathrm{o}}^{2} \mu} \sqrt{\frac{2 \rho}{p^{3}}} v_{c}$,

where $v_{c}$ is given by Eq. (7.7). The process-level energy efficiency for the AWJM process as a function of the pump pressure is plotted in Fig. 11. 


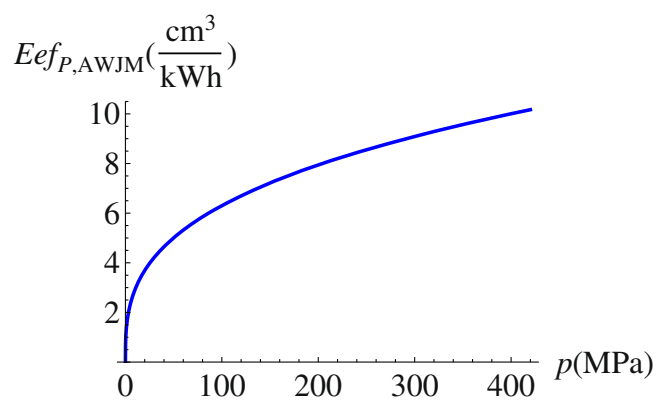

Fig. 11 The process-level energy efficiency for the AWJM process as function of the pump pressure

The time required for the AWJM process equals

$t_{\mathrm{AWJM}}=\frac{\pi\left(D-\frac{k}{2}\right)}{v_{c}}$.

The Eqs. (7.5), (7.6), (7.11), and (7.12) are the outcome of the energy efficiency study of the process level. As shown in Figs. 10 and 11, the process-level energy efficiency increases as the value of the relevant selectable process variable (feed rate or pump pressure) increases. This trend is stronger for smaller values of the process variable, while for larger values, the dependence of the process-level energy efficiency on the process variable value becomes weaker.

\subsection{Machine-level study}

At the machine level, any energy losses due to machine peripherals or machine inefficiencies have to be taken into account and at the same time, as the processes, under study, are removing processes, possible differences between the process-level "result" and the machine-level "result" have to be taken into account.

For the drilling process, the machine-level "result" is identical to the process-level "result" and is equal to

result $_{P, \text { drill }}=$ result $_{M, \text { drill }}=\pi \frac{D^{2}}{4} w$.

However, since the AWJM process is a trepanning process, the process- and machine-level "results" are different,

$$
\begin{aligned}
\operatorname{result}_{P, \mathrm{AWJM}} & =\frac{4 k(D-k)}{D^{2}} \operatorname{result}_{M, \mathrm{AWJM}} \\
& =\pi k(D-k) w .
\end{aligned}
$$

Moreover, the drill motor and the AWJM pump are characterized by efficiency $n_{\text {drill }}$ and $n_{\mathrm{AWJM}}$, respectively, and by peripherals power consumption $P_{\text {per,drill }}$ and $P_{\text {per,AWJM }}$. The values of the aforementioned parameters for the machines under study have been measured, and their values are given in Table 1.
Table 1 The efficiency and peripherals consumption for the two machines under study

\begin{tabular}{lll}
\hline Machine & Motor/pump efficiency & Peripherals consumption (W) \\
\hline Drill & 0.85 & 500 \\
AWJM & 0.76 & 500 \\
\hline
\end{tabular}

According to Section 4, the machine-level energy efficiency of the specific machines is

$$
\begin{aligned}
& E e f_{M, \text { drill }}=\frac{E e f_{P, \text { drill }}}{\frac{1}{n_{\text {drill }}}+\frac{P_{\text {per,drill }} t_{\text {drill }}}{E_{P, \text { drill }}}} . \\
& E e f_{M, \mathrm{AWJM}}=\frac{D^{2}}{4 k(D-k)} \frac{E e f_{P, \mathrm{AWJM}}}{\frac{1}{n_{\mathrm{AWJM}}}+\frac{P_{\text {per,AWJM }} t_{\mathrm{AWJM}}}{E_{P, \mathrm{AWM}}}} .
\end{aligned}
$$

The energy efficiency, at the machine level, as function of the relevant process variable is plotted in Figs. 12 and 13. For both processes and both holes, the maximum value of the relevant process variable is the most energy-efficient. This is expected since from the Eqs. (7.5), (7.6), (7.11), and (7.12), it can be shown that the former minimizes the process time and thus the peripherals consumption, simultaneously maximizing the process-level energy efficiency.

It has to be noted that using the maximum process variable value and comparing the energy efficiency of the two machines for holes of arbitrary diameter, the selection of the most energy-efficient machine is not obvious. This is due to the fact that when the hole diameter gets larger, the number of tool changes in the drilling process is increased. Each additional tool change corresponds to the process time being drastically increased, thus resulting in an increase of the energy consumption, due to the peripherals. This is shown in Fig. 14.

From Fig. 14, as well as the relations (7.15) and (7.16), it turns out that for the case studied, the drill is more energy efficient when it comes to the smaller hole, while the AWJM machine is more energy efficient for the larger hole. It has to be noted that at the process level, the drilling process

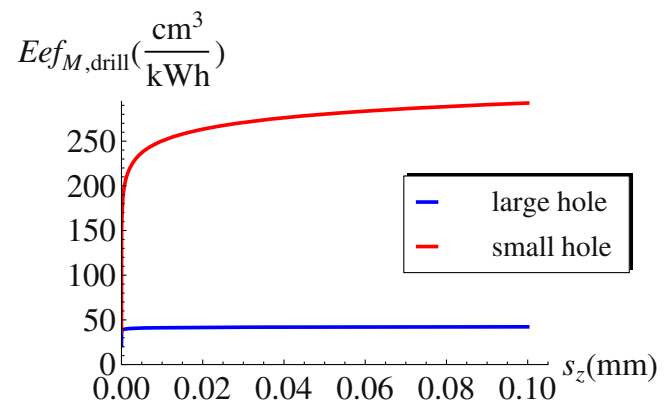

Fig. 12 The machine-level energy efficiency for the drill machine under study, as function of the feed rate per revolution and per tool edge 


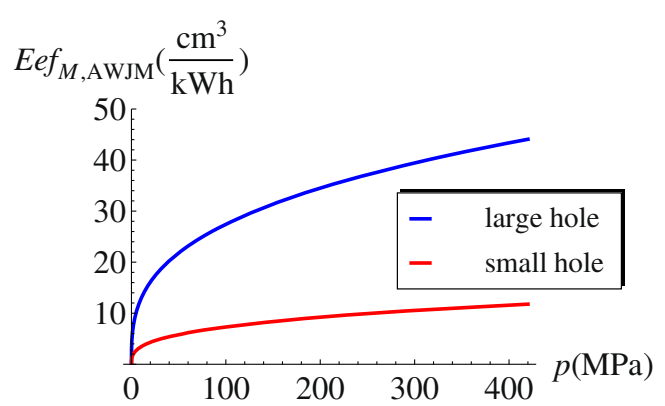

Fig. 13 The machine-level energy efficiency for the AWJM machine under study, as function of the pump pressure

appears more efficient than the AWJM process. However, for the large hole, due to the different process times, the AWJM is more efficient at the machine level due to smaller peripherals consumption. This is a consequence of energy efficiency inter-level interactions between the process and machine levels.

\subsection{Production line-level study}

As described in Section 7.1, the case study is about the making of two holes in a mild steel sheet, one of 4.9- $\mathrm{mm}$ diameter (small hole) and one of 22-mm diameter (large hole). Since two machines are available for each task, there are four possible production line configurations.

The machine-level energies required $\left(E_{M}\right)$, as well as the relevant process times $\left(t_{P}\right)$ for the opening of both holes, are shown in Table 2.

In the following,

$t_{\text {large }} \quad$ is the process time for the making of the large hole, $t_{\text {small }}$ is the process time for the making of the small hole,

$E_{\text {large }}$ is the energy required for the making of the large hole,

$E_{\text {small }}$ is the energy required for the making of the small hole,

Resource1 is the machine assigned for the making of the large hole,

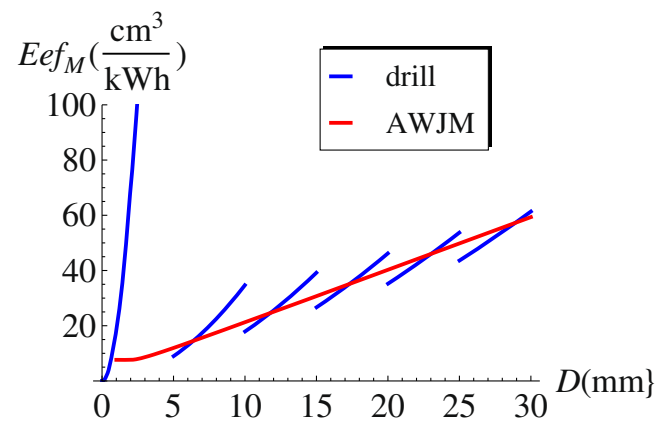

Fig. 14 Comparison of the machine-level energy efficiency for the drill and AWJM machine, as function of the hole diameter
Table 2 Machine-level energy consumptions and process times, if the optimum selection of process variables is made, for the two operations of the case study and the two machines considered

\begin{tabular}{llll}
\hline Operation & Machine & $t_{p}(\min )$ & $E_{M}(\mathrm{kWh})$ \\
\hline Large hole & Drill & 2.05 & 0.018 \\
Small hole & Drill & 0.01 & 0.00013 \\
Large hole & AWJM & 0.021 & 0.017 \\
Small hole & AWJM & 0.0040 & 0.0032 \\
\hline
\end{tabular}

Resource2 is the machine assigned for the making of the small hole, if different than Resource1,

$t_{\mathrm{idle}, 1}$ is the time period that Resource1 remains idle,

$t_{\mathrm{idle}, 2}$ is the time period that Resource 2 remains idle,

$t_{\text {mount }}$ is the required time for mounting the workpiece. The setup time $t_{\text {mount }}$ is on average equal to $0.5 \mathrm{~min}$ if the two holes are made by different machines and $0.25 \mathrm{~min}$ if the two holes are made by the same machine.

$P_{\text {per, } 1}$ is the Resource1 idle power consumption,

$P_{\text {per,2 }}$ is the Resource 2 idle power consumption,

$E_{\text {idle }} \quad$ is the overall (all resources) energy consumption during idle times per part produced.

The production schedule, which determines the idle times, is shown in Fig. 15. If $N$ workpieces are manufactured and two different machines are used, then the idle time for Resource1 equals $t_{\text {idle, } 1}=N t_{\text {mount }}$, while for Resource 2 equals $t_{\text {idle }, 2}=N\left(t_{\text {mount }}+t_{\text {large }}-t_{\text {small }}\right)$. If only one machine is used, then its idle time equals $t_{\text {idle }}=$ $2 N t_{\text {mount }}$.

Thus, the idle consumption per part is equal to $E_{\text {idle }}=$ $P_{\text {per }, 1} t_{\text {mount }}+P_{\text {per }, 2}\left(t_{\text {large }}-t_{\text {small }}+t_{\text {mount }}\right)$ if a two-machine

(a)

Resource1

Resource2

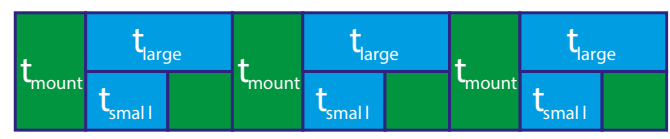

(b)
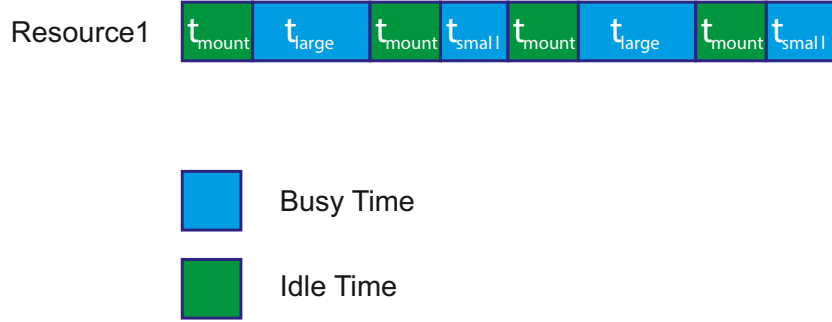

Fig. 15 Typical schedule for two-machine (a) and one-machine (b) configurations of the production line 


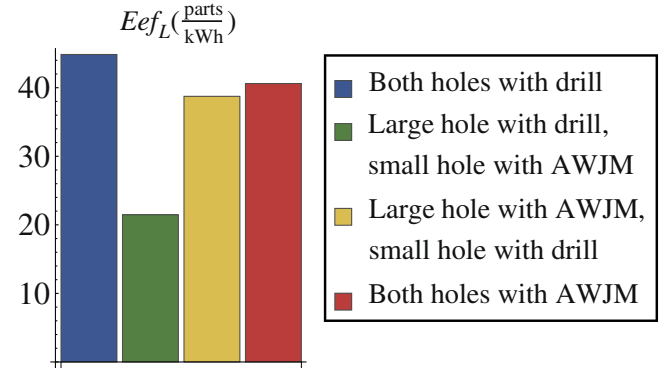

Fig. 16 Comparison of the production line energy efficiency for the different production line configurations

configuration is used, while it equals $E_{\text {idle }}=2 P_{\text {per, } 1} t_{\text {mount }}$ if an one-machine configuration is used.

According to the definition of production line-level energy efficiency $\left(\right.$ Eef $\left._{\mathrm{L}}\right)$, given in Section 5, the energy efficiency, at the production line level, equals

Eef $_{\mathrm{L}}=\frac{1}{E_{\text {large }}+E_{\text {small }}+E_{\text {idle }}}$.

The comparison of the four possible production line configurations is shown in Fig. 16 and Table 3.

Thus, according to Fig. 16, the most energy-efficient production line configuration is that of the drill for both holes, although the drill was less energy-efficient than the AWJM machine for the large hole, at the machine level.This happens due to inter-level interactions between the machine and production line levels.

\subsection{Discussion}

The drill becomes less and less efficient as the size of the hole to be drilled increases, as a result of the increase in the process time due to the necessary tool changes. This is evident in Fig. 12. The peripherals play a very important role to this process. The AWJM machine becomes more and more efficient as the size of the hole to be made increases, as shown in Fig. 13.

Although, at the process level, the drill is more efficient, peripherals' consumption makes the selection between the drill and the AWJM not obvious, as shown in Fig. 14. Additionally, while the AWJM machine is more efficient for the making of the large hole, at the machine level, machine's

Table 3 The production line-level energy efficiency for the different production line configurations

\begin{tabular}{ll}
\hline Configuration & $\operatorname{Eef}_{\mathrm{L}}(\mathrm{parts} / \mathrm{kWh})$ \\
\hline Both holes with drill & 44.8 \\
Large hole with drill, small hole with AWJM & 21.5 \\
Large hole with AWJM, small hole with drill & 38.7 \\
Both holes with AWJM & 40.6 \\
\hline
\end{tabular}

idle consumption results in the most energy-efficient production line configuration, being the drill used for both holes, as shown in Fig. 16.

The proposed strategy only considers the inter-level interactions between neighboring levels; for example, the influence of process variables through process time on the production line schedule is not taken into account. This simplification, however, reduces the complexity of the problem, as shown in Section 7.4, allowing the use of Table 2 for the production line-level analysis. It has to be reckoned that in more complex cases, the interaction of more machines or production lines with each other and with more criteria, such as cost or quality being taken into account, makes this simplification much more important.

The construction of a process-level energy efficiency library for all processes, such as Eqs. (7.5), (7.6), (7.11), and (7.12), as described in Section 3, will significantly simplify the application of the energy efficiency optimization strategy. Since these relations do not depend on the workpiece geometry or the machine characteristics, they can be used for the study of other production lines that include these processes as well.

\section{Conclusions}

The key question in manufacturing today is finding the way to produce more with less, by satisfying the world demand. It is clear that energy efficiency has to be included in the manufacturing decision-making attributes.

1. Energy efficiency has to be defined separately for each manufacturing level, since energy consumption is affected by a set of factors of quite divergent nature. This classification allows for the easier study of energy efficiency.

2. The energy efficiency definition provided in this paper, given in Eq. (2.1), can adapt to this classification.

3. The presence of nontrivial inter-level interactions does not allow for a level-by-level address of energy efficiency. A more complicated strategy is required, and it is described in Section 2.1.

4. Process-level energy efficiency is successfully defined for all manufacturing processes. The proper process "result" in these definitions is equal or proportional to the volume affected by the process. The generic nature of these definitions allows the construction of a library for each process, which will facilitate the study of energy efficiency at the higher levels.

5. Although the energy spent on the process itself is small, the appropriate selection of process variables may also alter the consumption of machine peripherals that correspond to a larger percentage of energy consumption 
(Fig. 6). For this reason, the study of the peripherals consumption as a function of the process variables is required for each machine.

6. Processing time is a function of the process variables. Thus, the selection of appropriate process variables has consequences for both the machine peripherals consumptions (machine level) and the production planning (line and factory levels).

7. In removing processes, the process-level "result" cannot be adopted as the machine-level "result." The machine-level energy efficiency definition has to be adjusted in a workpiece geometry-dependent way, so that the machines performing different manufacturing processes and yielding the same outcome will be comparable.

8. A large percentage of energy spent by a manufacturing industry is wasted on idle machine consumption. Energy efficiency at the production line and factory levels can be significantly improved with better scheduling and the inclusion of shutdown or eco-modes in the work planning of the machines.

Acknowledgments The work reported in this paper was supported by the collaborative program entitled "Energy Efficient Process planning System ENEPLAN," which is under the seventh framework program - FoF.NMP.2011-1: The Eco-Factory: cleaner and more resource-efficient production in manufacturing Program.

\section{References}

1. Chryssolouris G (2006) Manufacturing systems: theory and practice, 2nd edn. Springer, New York

2. Chryssolouris G, Papakostas N, Mavrikios D (2008) A perspective on manufacturing strategy: produce more with less. CIRP J Manuf Sci Technol 1:45-52

3. Gutowski T, Dahmus J, Thiriez A (2006) Electrical energy requirements for manufacturing processes. In: 13th CIRP international conference on life cycle engineering

4. Duflou JR, Sutherland JW, Dornfeld D, Herrmann C, Jeswiet J, Kara S, Hauschild M et al (2012) Towards energy and resource efficient manufacturing: a processes and systems approach. CIRP Ann Manuf Technol 61:587-609

5. Int. Energy Agency (IEA) (2008) Worldwide trends in energy use and efficiency, key insights from IEA indicator analysis. http://www.iea.org/publications/freepublications/publication/ Indicators_2008-1.pdf. Accessed 18 Sep 2013

6. EIA (2010) Annual energy review 2010:38 http://www.eia. gov/totalenergy/data/annual/archive/038410.pdf. Accessed 18 Sep 2013

7. Bay N, Azushima A, Groche P, Ishibashi I, Merklein M, Morishita M, Nakamura $T$ et al (2010) Environmentally benign tribosystems for metal forming. CIRP Ann Manuf Technol 59:760780

8. Li C, Jiang S, Zhang K (2012) Pulse current-assisted hot-forming of light metal alloy. Int J Adv Manuf Technol 63:931-938

9. Duflou JR, Kellens K, Guo Y, Dewulf W (2012) Critical comparison of methods to determine the energy input for discrete manufacturing processes. CIRP Ann Manuf Technol 61:63-66
10. Kara S, Li W (2011) Unit process energy consumption models for material removal processes. CIRP Ann Manuf Technol 60:37-40

11. Mori M, Fujishima M, Inamasu Y, Oda Y (2011) A study on energy efficiency improvement for machine tools. CIRP Ann Manuf Technol 60:145-148

12. Oda Y, Mori M, Ogawa K, Nishida S, Fujishima M, Kawamura $\mathrm{T}$ (2012) Study of optimal cutting condition for energy efficiency improvement in ball end milling with tool-workpiece inclination. CIRP Ann Manuf Technol 61:119-122

13. Schlosser R, Klocke F, Lung D (2011) Sustainability in manufacturing - energy consumption of cutting processes. In: Advances in sustainable manufacturing. Springer, Heidelberg, pp 85-89

14. Li W, Winter M, Kara S, Herrmann C (2012) Eco-efficiency of manufacturing processes: a grinding case. CIRP Ann Manuf Technol 61:59-62

15. Kellens K, Dewulf W, Duflou JR (2011) Preliminary environmental assessment of electrical discharge machining. In: Proceedings of the 18th CIRP international conference on life cycle engineering. doi:10.1007/978-3-642-19692-8_65

16. Somashekhar KP, Ramachandran N, Mathew J (2010) Material removal characteristics of microslot (kerf) geometry in -WEDM on aluminum. Int J Adv Manuf Technol 51:611-626

17. Fysikopoulos A, Stavropoulos P, Salonitis P, Chryssolouris G (2012) Energy efficiency assessment of laser drilling process. Phys Procedia 39:776-783

18. Al-Sulaiman F, Yilbas B, Karakas F, Ahsan M, Mokheimer E (2008) Laser hole cutting in Kevlar: modeling and quality assessment. Int J Adv Manuf Technol 38:1125-1136

19. Behrendt T, Zein A, Min S (2012) Development of an energy consumption monitoring procedure for machine tools. CIRP Ann Manuf Technol 61:43-46

20. Vijayaraghavan A, Dornfeld D (2010) Automated energy monitoring of machine tools. CIRP Ann Manuf Technol 59:21-24

21. Huang B, Xing K, Abhary K, Spuzic S (2012) Development of energy-saving optimization for the oval-edging oval pass design using genetic algorithm. Int J Adv Manuf Technol 61:423-429

22. Göschel A, Schieck F, Schönherr J (2012) Method for energy and resource balancing demonstrated as an example of the hot sheet metal production process. CIRP Ann Manuf Technol 61:399-402

23. Helu M, Behmann B, Meier H, Dornfeld D, Lanza G, Schulze V (2012) Impact of green machining strategies on achieved surface quality. CIRP Ann - Manuf Technol 61:55-58

24. Bruzzone AAG, Anghinolfi D, Paolucci M, Tonelli F (2012) Energy-aware scheduling for improving manufacturing process sustainability: a mathematical model for flexible flow shops. CIRP Ann - Manuf Technol 61:459-462

25. Weinert N, Chiotellis S, Seliger G (2011) Methodology for planning and operating energy-efficient production systems. CIRP Ann - Manuf Technol 60:41-44

26. Herrmann C, Thiede S, Kara S, Hesselbach J (2011) Energy oriented simulation of manufacturing systems - concept and application. CIRP Ann - Manuf Technol 60:45-48

27. Herrmann C, Thiede S (2009) Process chain simulation to foster energy efficiency in manufacturing. CIRP J Manuf Sci Technol 1:221-229

28. Harms R, Fleschutz T, Seliger G (2010) Life cycle management of production facilities using semantic web technologies. CIRP Ann Manuf Technol 59:45-48

29. Kellens K, Dewulf W, Overcash M, Hauschild MZ, Duflou JR (2011) Methodology for systematic analysis and improvement of manufacturing unit process life-cycle inventory (UPLCI)-CO2PE! initiative (cooperative effort on process emissions in manufacturing). Part 1: methodology description. Int $\mathbf{J}$ Life Cycle Assess 17:69-78 
30. Umeda Y, Takata S, Kimura F, Tomiyama T, Sutherland JW, Kara S, Herrmann C et al (2012) Toward integrated product and process life cycle planning-an environmental perspective. CIRP Ann Manuf Technol 61:681-702

31. Papakostas N, Efthymiou K, Mourtzis D, Chryssolouris G (2009) Modeling the complexity of manufacturing systems using nonlinear dynamics approaches. CIRP Ann - Manuf Technol 58:437440

32. Chryssolouris G, Efthymiou K, Papakostas N, Mourtzis D, Pagoropoulos A (2013) Flexibility and complexity: is it a tradeoff? Int J Prod Res 51:6788-6802. doi:10.1080/00.2075432012. 761362

33. Fysikopoulos A, Papacharalampopoulos A, Pastras G, Stavropoulos P, Chryssolouris G (2013) Energy efficiency of manufacturing processes: a critical review. Procedia CIRP 7:628-633

34. Hon K. K. B (2005) Performance and evaluation of manufacturing systems. CIRP Ann - Manuf Technol 54:139-154

35. Efthymiou K, Pagoropoulos A, Papakostas N, Mourtzis D, Chryssolouris G (2012) Manufacturing systems complexity review: challenges and outlook. Procedia CIRP 3:734-743

36. Patterson MG (1996) What is energy efficiency? Concepts, indicators and methodological issues. Energy Policy 24(5):377-390

37. Wang Q, Liu F, Li C (2013) An integrated method for assessing the energy efficiency of machining workshop. J Clean Prod 52:122133

38. Cohen ER, Cvitas T, Frey JG, Holmstrm B, Kuchitsu K et al (2008) Quantities, units and symbols in physical chemistry. IUPAC green book, 3rd edn. IUPAC \& RSC Publishing, Cambridge 6

39. Gutowski T, Dahmus J, Thiriez A, Branham M, Jones A (2007) A thermodynamic characterization of manufacturing processes. In: Proceedings of the 2007 IEEE international symposium on electronics and the environment. doi:10.1109/ISEE.2007.369382

40. Bakshi B, Gutowski TG, Sekulic DP (2011) Thermodynamics and the destruction of resources. Cambridge University Press, New York, pp 163-189

41. Renaldi, Kellens K, Dewulf W, Duflou JR (2012) On the implementation of energy efficiency metrics in discrete manufacturing system: the dissipative nature of production processes. Leveraging Technology for a Sustainable World. In: Proceedings of the 19th CIRP conference on life cycle engineering, pp 545-550

42. Fysikopoulos A, Anagnostakis D, Salonitis K, Chryssolouris G (2012) An empirical study of the energy consumption in auto- motive assembly. In: 45th CIRP conference on manufacturing systems, pp 540-547

43. Gutowski T, Murphy C, Allen D, Bauer D, Bras B, Piwonka $\mathrm{T}$, Sheng $\mathrm{P}$ et al (2005) Environmentally benign manufacturing: observations from Japan, Europe and the United States. J Clean Prod 13:1-17

44. Devoldere T, Dewulf W, Deprez W, Willems B, Duflou J (2007) Improvement potential for energy consumption in discrete part production machines. In: Proceedings of 14th CIRP conference on LCE, pp 311-316

45. Kahlenborn W, Kabisch S, Klein J, Richter I, Schrmann S (2010) DIN EN 16001: energy management systems in practice - a guide for companies and organisations. Bundesministerium fr Umwelt, Naturschutz und Reaktorsicherheit, Berlin, Germany

46. ENEPLAN Project. http://www.eneplan.eu. Accessed 18 Sep 2013

47. Mouzon G, Yildirim MB, Twomey J (2007) Operational methods for minimization of energy consumption of manufacturing equipment. Int J Prod Res 45:4247-4271

48. Mouzon G (2008) Operational methods and models for minimization of energy consumption in a manufacturing environment. Dissertation, Wichita State University

49. Glock CH (2012) The joint economic lot size problem: a review. Int J Prod Econ 135:671-686

50. Müller E, Löffler T (2010) Energy efficiency at manufacturing plants-a planning approach. In: 43rd CIRP international conference on manufacturing systems, pp 787-794

51. Chryssolouris G, Pierce J, Dicke K (1992) A decision-making approach to the operation of flexible manufacturing systems. Int J Flex Manuf Syst 4:309-330

52. Chryssolouris G, Subramaniam V (2001) Dynamic scheduling of manufacturing job shops using genetic algorithms. J Intell Manuf $12: 281-293$

53. European Commission (2009) CT and energy efficiency the case for manufacturing. http://ec.europa.eu/information_society/ events/ict4ee/2009/docs/files/ec/ec/infso/g2/SmartManufacturing. pdf Accessed 18 Sep 2013

54. Tschätsch H (2009) Applied machining technology. Springer, New York. ISBN 978-3-642-01006-4

55. Zeng J, Olsen J, Olsen C (1999) The abrasive water jet as a precision metal cutting tool. In: Proceedings of the 10th American water jet conference, pp 829-843 